\title{
Imaging Alzheimer in 2011
}

\author{
Marieke Hazewinkel • Frederik Barkhof
}

(C) Springer-Verlag 2011

Over the past two decades, MRI has come to play a prominent role in the diagnosis of Alzheimer Dementia (AD). Findings that have a high sensitivity for AD are medial temporal lobe atrophy (Fig. 1) and parietal atrophy (Fig. 2), especially when both are present in the same patient. If MRI is contraindicated or not available, CT is a good alternative with which global and medial temporal lobe atrophy as well as the extent of white matter lesions may be reliably assessed.

Medial temporal lobe atrophy (MTA) on MRI is now considered to be one of the three diagnostic criteria for progression to $\mathrm{AD}$ in patients with mild cognitive impairment (MCI), along with temporoparietal hypometabolism on FDG-PET and tau/amyloid- $\beta$ in CSF.

Imaging studies have made a large contribution to insights in how the disease develops. Pathologically, AD is characterized by the deposition and progressive build-up of abnormal proteins (amyloid- $\beta$ and hyper-phosphorylated tau) in the brain, which interfere with normal function and cause neuronal and axonal loss. These proteins form plaques and tangles, initially only affecting the temporal lobes and limbic system, later spreading to the neocortex. Atrophy patterns in AD mirror this pattern of abnormal protein deposition, only at a much later stage in the disease.

Amyloid- $\beta$ can be detected in vivo using PET using amyloid-binding compounds such as the ${ }^{11} \mathrm{C}$-labelled Pittsburgh Compound-B (PiB) or ${ }^{18} \mathrm{~F}$-fluorinated variants thereof; the latter have longer decay-times and will be more suitable for clinical applications (Fig. 3). These abnormal proteins which accumulate in the brains of Alzheimer patients may be present up to 30 years before the manifestation of any

M. Hazewinkel · F. Barkhof $(\varangle)$

Afdeling Radiologie, Academisch Ziekenhuis, Vrije Universiteit,

De Boelelaan 1117, Postbus 7057, Amsterdam, The Netherlands

e-mail: f.barkhof@vumc.nl

F. Barkhof

e-mail: R.Wijhenke@vumc.nl clinical symptoms, and may also be found in normal older subjects who do not develop dementia. The presence of these proteins alone are therefore not sufficient for the diagnosis. Similarly, finding only an abnormally low CSF amyloid level may not have clinical relevance in the absence of increased tau (or abnormal MRI).

FDG-PET is another tool which is used in the diagnosis of AD. Patients with AD typically show hypometabolism in the temporoparietal, frontal and posterior cingulate cortices and hippocampus. These findings are highly sensitive $(>90 \%)$, but unfortunately not so specific for AD. It can not always discriminate between $\mathrm{AD}$ and other forms of dementia such as frontotemporal dementia (FTLD) and dementia with Lewy bodies (DLB). These areas of hypometabolism may also be seen in patients with $\mathrm{MCI}-$ with good sensitivity (86\%) and specificity (96\%) in differentiating these patients from normal subjects. FDGPET could therefore be one modality useful in identifying those patients with MCI that will progress to develop dementia. Not only is this valuable information for the patients and their families, it is also becoming increasingly important to identify early stages of dementia in the light of potential disease-modifying drugs. Arterial spin-labelling (ASL) is an upcoming MR technique that may provide similar information about temporal-parietal hypoperfusion, without the need of injecting a radio-active tracer

MRI is a useful diagnostic tool in that not only aids in discriminating different forms of dementia, it is also helpful in identifying which patients with MCI are likely to develop $\mathrm{AD}$. As referred to earlier, medial temporal lobe and parietal atrophy have a positive predictive value in the diagnosis of AD. In clinical practice, MTA may be assessed using a visual scale from 0 to 4 . Cortical atrophy may be visually assed using the Global Cortical Atrophy Scale (0-3). Discordant parietal atrophy, particularly of the precuneus, is also characteristic of $\mathrm{AD}$, especially in the presenile variant when medial temporal lobe atrophy may not be present. MR images of patients with $\mathrm{AD}$ 


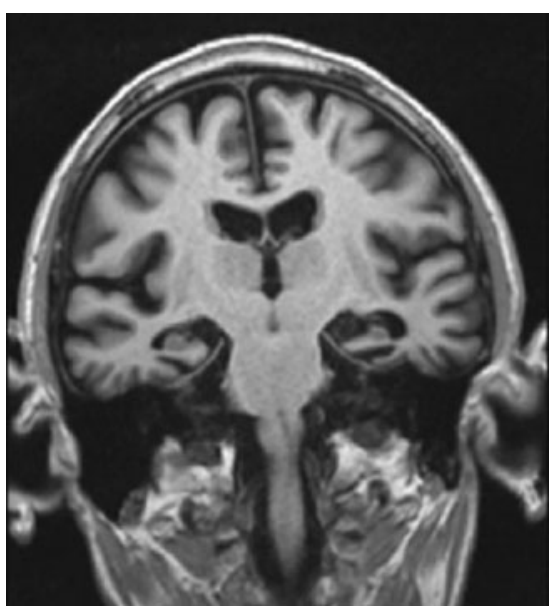

Fig. 1 Coronal T1-weighted image showing medial temporal lobe atrophy, with profound volume loss on the left

Fig. 2 Sagittal and coronal T1-weighted images of an early-onset AD patient with precuneus/parietal atrophy (left). Note that there is no medial temporal lobe atrophy (right)

Fig. 3 Positive (left) and negative (right) PiB-PET studies with classic AD frontotemporal uptake on the left

Table 1 Overview of findings on MRI and molecular imaging in differentiating $\mathrm{AD}$ from FTLD and DLB
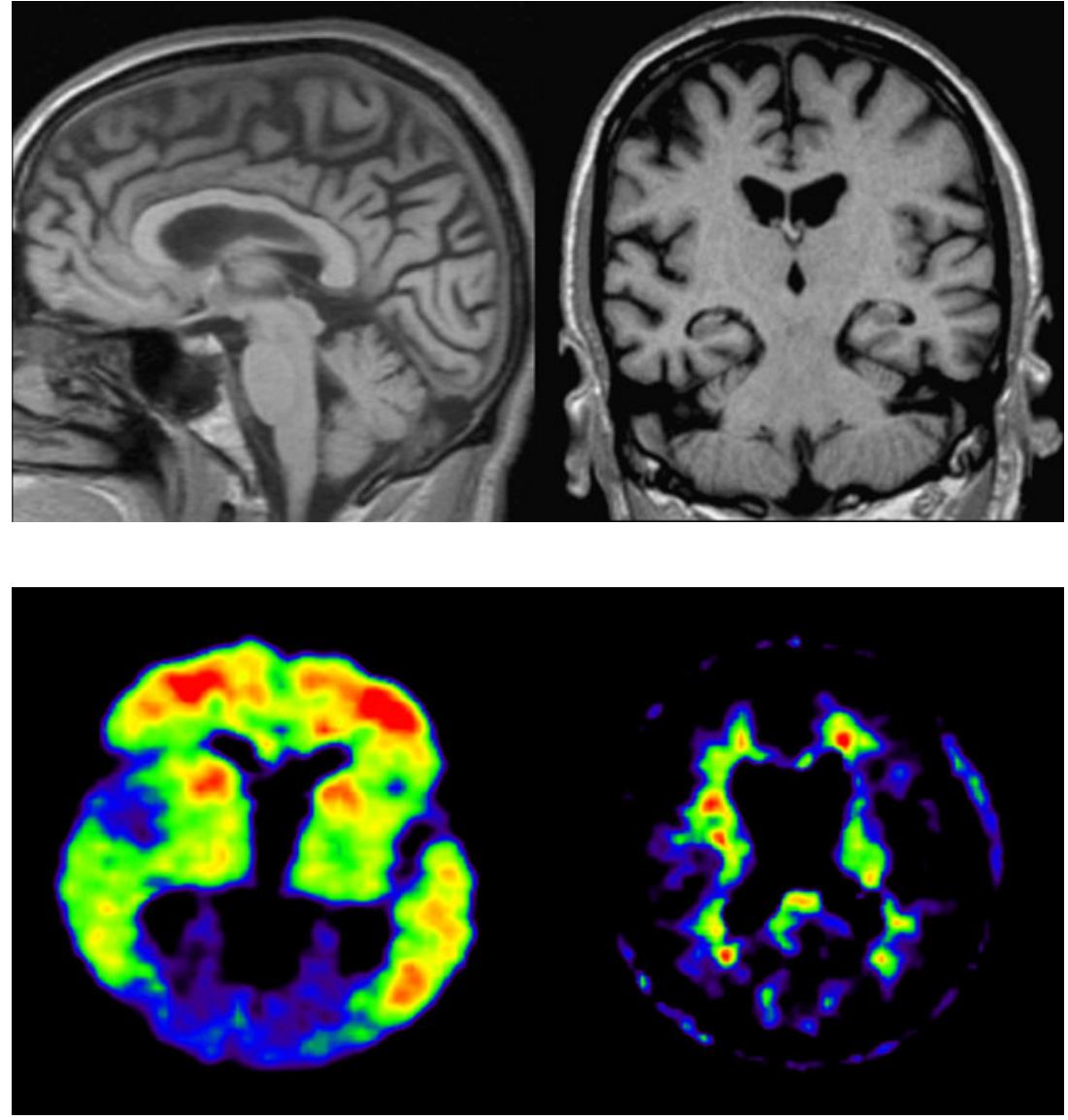

\begin{tabular}{lllll}
\hline & MRI & FDG hypometabolism & PiB binding & Dopamine SPECT \\
\hline AD & $\begin{array}{c}\text { medial temporal lobe, } \\
\text { parietal atrophy } \\
\text { (asymmetric) frontal and/or } \\
\text { temporal atrophy } \\
\text { parietal and/or occipital atrophy }\end{array}$ & $\begin{array}{c}\text { frontotemporal and } \\
\text { posterior cingulum } \\
\text { frontal }\end{array}$ & yes & no \\
DLB & occipital & possible & $\begin{array}{c}\text { normal } \\
\text { decreased binding } \\
\text { basal ganglia }\end{array}$ \\
\hline
\end{tabular}


Fig. 4 Theoretical model demonstrating cognitive and biological markers in the natural progression of $\mathrm{AD}$ (reprinted with permission from Frisoni, G. B. et al. Nat. Rev. Neurol. 2010:6, 67-77)

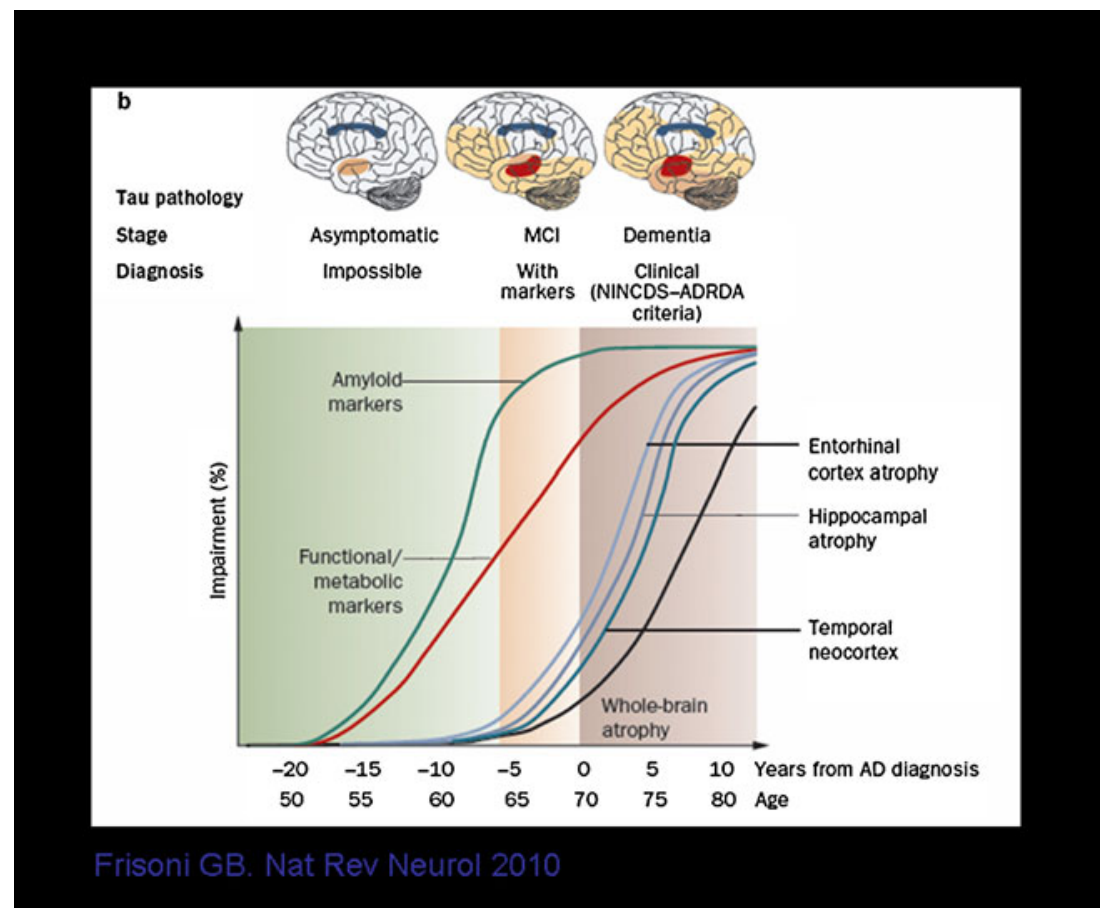

may also show white matter hyperintensities and microbleeds. The main differentials of AD are FTLD and DLB, with partially overlapping MRI features. Table 1. provides an overview of how these may be differentiated using additional (molecular) imaging. Figure 4 shows a theoretical model of the natural progression of cognitive and biological markers in $\mathrm{AD}$.

\section{Suggested Reading}

1. Frisoni GB et al (2010) Nat. Rev Neurol 6:67-77

2. Braak H, Braak E (1991) Acta Neuropathol (Berl) 82:239-259

3. Jack et al (2010) Brain 133:3336-3348

4. Mosconi L et al (2008) J Nucl Med 49:390-398

5. Barkhof F, Fox N, Bastos-Leite A, Scheltens P (2011) Neuroimaging in Dementia. Springer 